\title{
Acute Presentation of Laparoscopic Gastric Band Erosion
}

\author{
Mohammed A. Bawahab, MD, FRCS (Canada), FASMBS \\ Department of Surgery, Faculty of Medicine, King Khalid, University, Abha, Saudi Arabia.
}

\begin{abstract}
Laparoscopic adjustable gastric band (LAGB) is a well-established bariatric surgical procedure. LAGB surgery is associated long term with a significant chance for reoperation within 10 years of band placement; 2 causes of reoperation are band migration and erosion. LAGB migration is a common late complication of gastric band, with a reported incidence of as much as $11 \%$. Herein we present a patient with LAGB erosion presenting as small bowel obstruction. In our patient, the LAGB had completely eroded into the stomach and migrated through the pylorus to the upper jejunum, resulting in a small bowel obstruction. We describe our laparoscopic approach and management of this complication, which requires a high index of suspicion.
\end{abstract}

Key Words: Obesity, Laparoscopic adjustable gastric band (LAGB), Erosion, Complication, Small bowel obstruction.

Citation Acute Presentation of Laparoscopic Gastric Band Erosion. Bawahab MA. CRSLS e2013.00244. DOI: 10.4293/CRSLS.2013.00244.

Copyright (C) 2015 by SLS, Society of Laparoendoscopic Surgeons. This is an open-access article distributed under the terms of the Creative Commons Attribution-Noncommercial-ShareAlike 3.0 Unported license, which permits unrestricted noncommercial use, distribution, and reproduction in any medium, provided the original author and source are credited.

Address correspondence to: Mohammed A. Bawahab, Chairman and Assistant Professor of General Surgery, General Surgery Department, Faculty of Medicine, King Khalid University, PO Box 641, Abha, Saudi Arabia 61421. Telephone: (009) 6650-575-5282, Fax: (009) 6617-2418-658. E-mail: bawahab1@hotmail.com.

\section{INTRODUCTION}

Morbid obesity is a significant medical problem that has negative effects on the general health of a person because of its association with major health problems such as diabetes, hypertension, heart disease, sleep apnea, and degenerative skeletal disorders. ${ }^{1}$ Metabolic surgery has been shown to be the most effective treatment to maintain consistent long-term weight loss in morbidly obese patients. Recently, patients with evidence of the metabolic syndrome and type 2 diabetes mellitus have been shown to benefit from undergoing bariatric surgery.

Bariatric surgical procedures are categorized into three types: restrictive, malabsorptive, and combined. Placement of a laparoscopic adjustable gastric band (LAGB) is one of the most frequently performed restrictive bariatric surgeries around the world. ${ }^{2}$ Its wide use is attributed to its simplicity, both in concept and procedure, and the procedure's complete reversibility. ${ }^{3}$ However, because of the high risk for reoperation and weight regain, LAGB has lost its appeal for both patients and surgeons. The rate of complications after LAGB placement cannot be ignored, despite their low numbers compared with those of other, more complex bariatric procedures. The complications that have been reported after LAGB are band slippage, band erosion, migration, pouch dilatation, infection at the port site, leakage, and outlet obstruction. ${ }^{3-7}$ In our case, we present a patient presenting with small bowel obstruction secondary to LAGB that eroded into the stomach and migrated through the pylorus to the upper jejunum, resulting in a small bowel obstruction.

\section{CASE REPORT}

A 20 -year-old man with a body mass index of $51.73 \mathrm{~kg} / \mathrm{m}^{2}$ (weight $322 \mathrm{lbs}$ ) and symptomatic osteoarthritis underwent LAGB via a perigastric approach in 2004. At the time of surgery, a LAP-BAND APS (10 mL; Allergan, Inc., Irvine, California) was placed. The immediate postoperative period was uneventful. The patient had satisfactory weight loss and was seen in 2 postoperative outpatient follow-up visits but was eventually lost to follow-up.

Four years later, the patient had maintained excellent weight loss, with a lowered body mass index of 22.68 $\mathrm{kg} / \mathrm{m}^{2}$ (weight $141 \mathrm{lbs}$ ). However, the patient presented with recurrent episodes of small bowel obstruction manifesting with intermittent attacks of colicky abdominal pain and vomiting that were treated conservatively by his 
primary health care providers on several occasions. Three months later after his initial presentation with complications, he presented to the emergency department with complete small bowel obstruction (persistent, severe colicky abdominal pain, bilious vomiting, abdominal distention, and obstipation) that had lasted 2 days. After initial fluid resuscitation, an erect plain abdominal radiograph was taken and showed the LAGB located in the middle of the abdomen in an unusual location, as well as signs of small bowel obstruction (Figure 1).

A computed tomography scan of the abdomen with intravenous and oral contrast was done (Figure 2) and revealed that the LAGB was located in the right lower quadrant of the abdomen; intraluminal migration of the LAGB causing small bowel obstruction was suspected. The patient underwent an upper gastrointestinal endoscopy, which revealed signs suspicious for LAGB erosion because the connecting tube of the LAGB was seen inside the stomach traversing the pylorus and duodenum.

After adequate fluid resuscitation, the decision was made to take the patient to surgery. During surgery, laparoscopic evaluation revealed that the LAGB had migrated

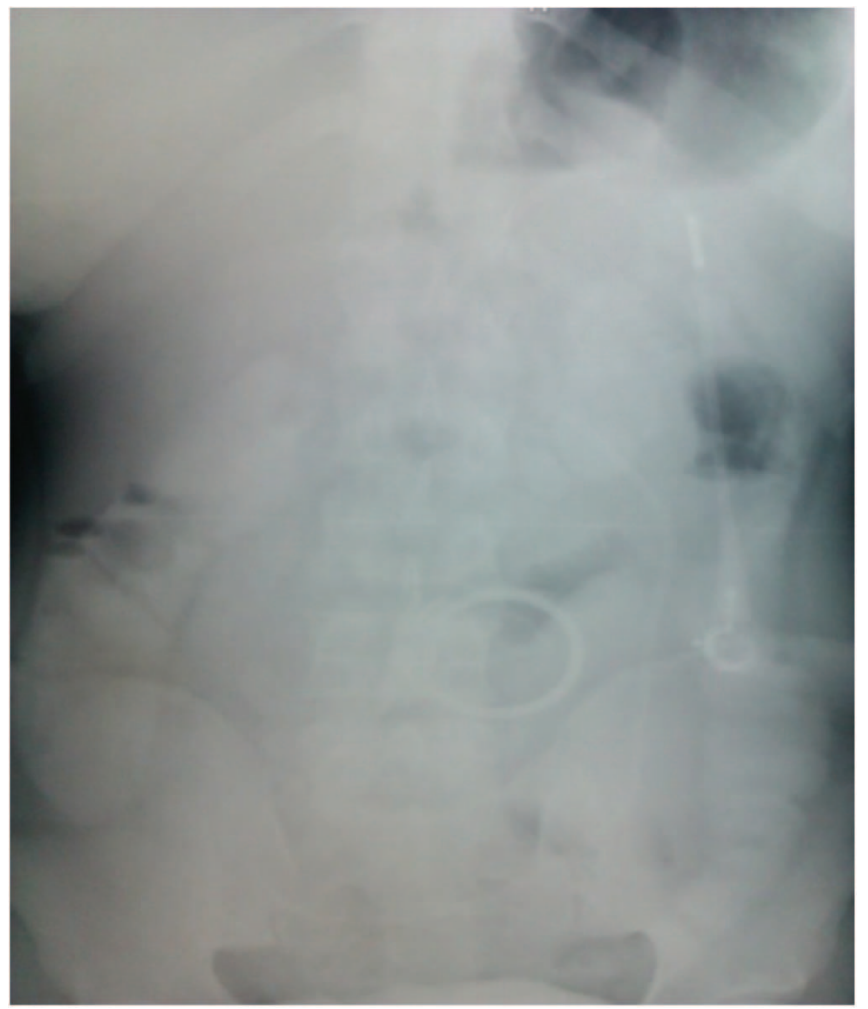

Figure 1. Plain radiograph of the abdomen taken in the erect position. intraluminally and caused a small bowel obstruction in the midjejunum. First, the connecting tube was divided just below the abdominal wall before its entrance to the gastric fundus, and we irrigated the small bowel carefully until the area of obstruction was found. Then a small laparotomy incision was made and the small bowel was pulled through the incision (Figure 3). Next, a small enterotomy was created and the LAGB was extracted (Figure 4) followed by suturing of the enterotomy by a single layer of absorbable interrupted sutures. The patient had a smooth postoperative course and was started on oral fluids on the second postoperative day. The patient tolerated a regular diet by the third day and was discharged in good condition.

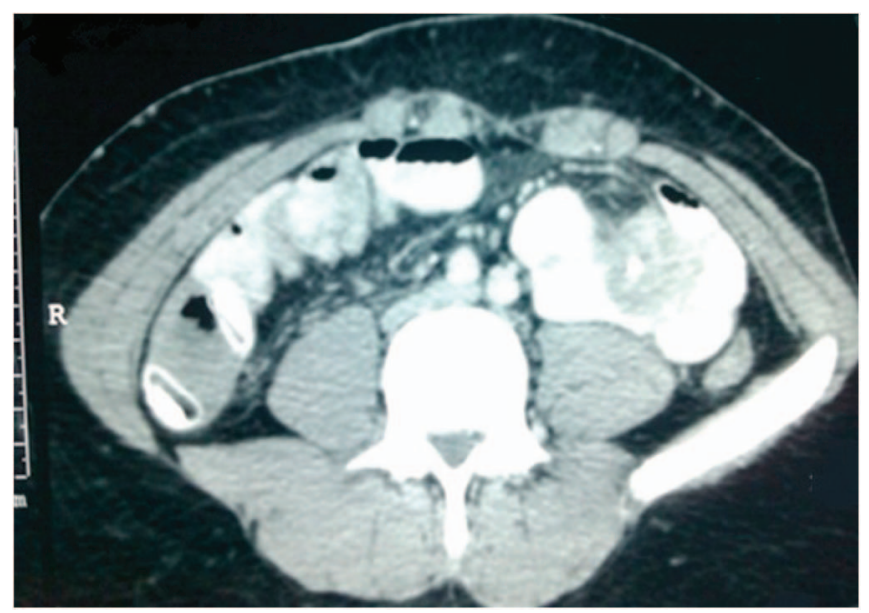

Figure 2. Computed tomography scan of abdomen with intravenous and oral contrast showing the band in the right lower quadrant.

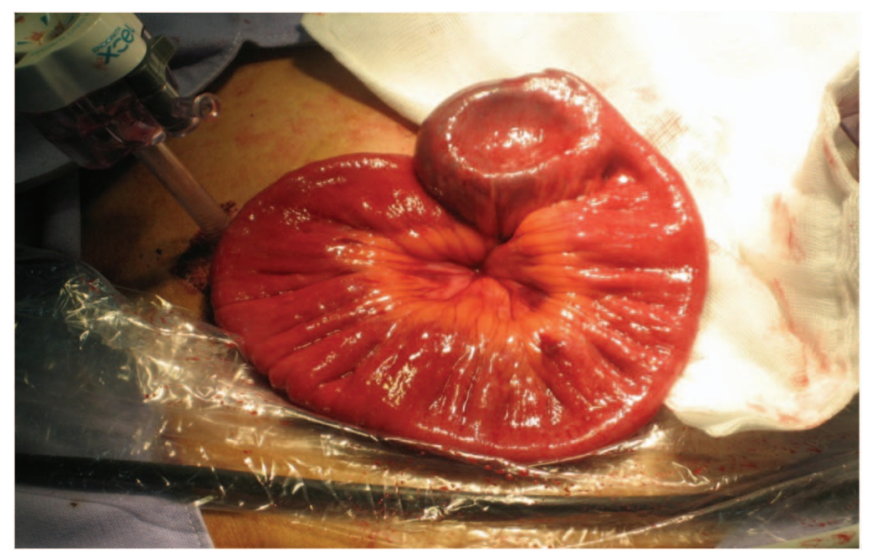

Figure 3. Extraction of the jejunal loop containing the lap band. 


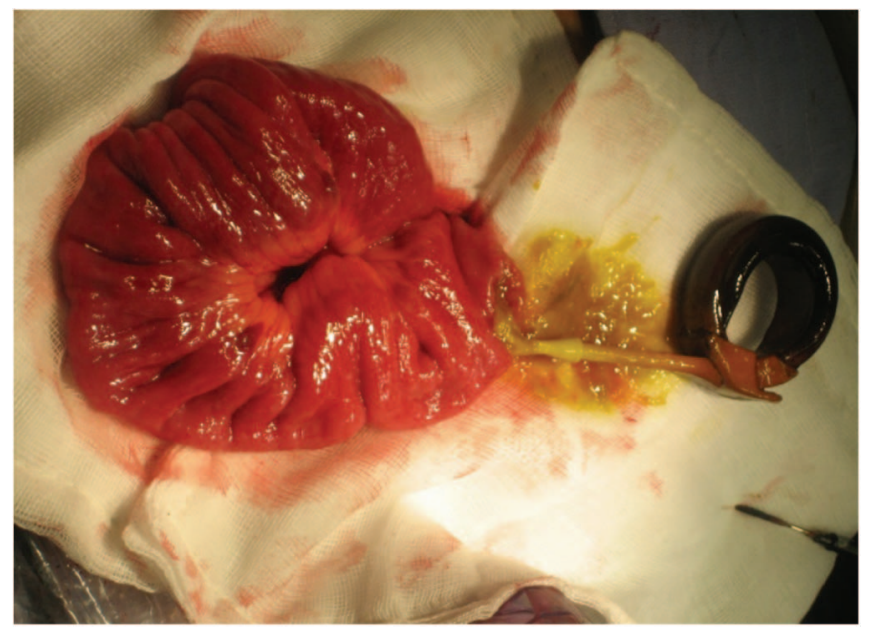

Figure 4. Band extraction through a small enterotomy.

\section{DISCUSSION}

LAGB is the safest bariatric procedure in the short term, but it can result in many complications that necessitate reoperation in the long term. The incidence of complications varies greatly between various series of cases. ${ }^{5-7}$ Nevertheless, the rate of complications observed is generally decreasing with improvement in the learning curve. ${ }^{7}$ Among the later complications noted is LAGB erosion, with an incidence varying between $0.6 \%$ and $11 \%$, that ultimately requires extraction of the LAGB. ${ }^{5}$ LAGB erosion can be silent and rarely causes peritonitis, but it should be suspected in any patient with unexplained weight gain after initial weight loss or if wound infection develops at the port site. . $^{5-6}$

The causes for LAGB erosion are poorly understood, but many recent theories explain why it is a less common complication compared with the initial LAGB procedures. Meir and Van Baden ${ }^{8}$ suggested four different etiologic hypotheses for LAGB erosion: (1) Abnormal reaction of the periprosthesic tissue in response to the presence of the LAGB, (2) infection of the band site, (3) excessive inflation of the band and consequent ischemia of the gastric wall, and (4) damage of the gastric wall during band implantation (caused by direct mechanical injury or a diathermy injury from electrocautery). In addition, LAGB erosion may be caused by placement of the LAGB using the old perigastric technique or using the old high-pressure LAGB similar to the one used in our patient. It is suggested that patients with gastric ulcers and those taking aspirin or nonsteroidal antiinflammatory drugs can be subjected to a greater risk of LAGB erosion as well. This was also observed in another case series in which patients with uncontrolled intake of nonsteroidal antirheumatic agents, broncho-spasmolytic drugs, and anticoagulant drugs showed a higher incidence of LAGB erosion. ${ }^{5}$

Furthermore, Lattauda et al analyzed fragments of fibroadipose tissue in close contact with the LAGB that were obtained at the time of band removal, suggesting that LAGB erosion could have a closer correlation with causes such as infection of the band or intraoperative surgical damage rather than with the specific material used to manufacture the band. ${ }^{?}$

According to Forsell et al, overfilling the LAGB (on average $12.6 \mathrm{~mL}$ in the "band migration group" in their series) may lead to pressure-induced erosion of the band through the gastric wall. ${ }^{10}$ The tighter the band, the greater the force exerted on the gastric wall in direct contact with the band, which causes excessive gastric wall pressure, leading to tissue ischemia and eventual erosion. ${ }^{11}$

The method by which the LAGB is placed can play a role in the incidence of LAGB erosion. There are two known techniques for dissection before LAGB placement: (1) The traditional perigastric pathway that requires dissection between the lesser curvature of stomach and lesser omentum, across the apex of the lesser sac, to the angle of His; and (2) the more recent technique of using the Pars flaccida pathway, in which dissection is made from the base of the right crus, along the left crus to the angle of His. O'Brien et $\mathrm{al}^{12}$ found that the using the Pars flaccida pathway is as effective as using the perigastric pathway in generating substantial weight loss, improved health, and improved quality of life, and it is significantly less likely to be associated with prolapse (slippage). Although the presented data suggest as a general recommendation that surgeons should prefer the Pars flaccida approach, Di Lorenzo et $\mathrm{al}^{13}$ suggested that the perigastric technique must be considered because in expert hands, it has good results as well, with a low complication rate. Thus the choice between using the Pars flaccida or perigastric pathway is linked to surgeon experience and strategy in the treatment of complications.

Laparoscopic omental plugging or gastric suturing was suggested in some studies after band removal. We believe this is not needed after laparoscopic band removal. In our patient, we did not repair the stomach after band removal in the reported case, and gastric fistula did not occur. Our opinion is supported by Mozzi et al, ${ }^{14}$ who suggested that gastric leak should be anticipated after surgical anterior gastrostomy and not after band removal. 
Band migration was reported in many previous studies. Cherian et $\mathrm{al}^{15}$ reported 17 cases of band erosion among 865 patients, 15 of which occurred relatively early in the series. Mittermair et al studied 733 patients, ${ }^{16}$ and band migration represented $6.5 \%$ of the cases. Kohn el al ${ }^{17}$ found 53 cases of gastric band erosion in 2097 patients studied. Most of the studies highlighted the decrease in the incidence of complications, including the band migration and erosion, with increased surgeon experience. Intragastric band migration constitutes the most common site in reported cases. The small intestine as a point of migration, as reported in our case, is considered a very rare site. Also the presentation of gastric band migration by intestinal obstruction is considered an extremely rare complication.

\section{CONCLUSION}

LAGB erosion and migration into the small intestine should be suspected in a patient with a history of LAGB and small bowel obstruction. Laparoscopic management of this complication is safe and feasible.

\section{References:}

1. Li Z, Bowerman S, Heber D. Health ramifications of the obesity epidemic. Surg Clin North Am. 2005;85:681-701.

2. Buchwald H, Oien DM. Metabolic/bariatric surgery worldwide 2008. Obes Surg. 2009;19:1605-1611.

3. Lanthaler M, Aigner F, Kinzl J, et al. Long-term results and complications following adjustable gastric banding. Obes Surg. 2010;20:1078-1085.

4. Steffen R, Biertho L, Ricklin T, Piec G, Horber F. Laparoscopic Swedish adjustable gastric banding: a five-year prospective study. Obes Surg. 2003;13:404-411.

5. Stroh C, Hohmann U, Will U, et al. Experiences of two centers of bariatric surgery in the treatment of intragastral band migration after gastric banding - the importance of the German multicenter observational study for quality assurance in obesity surgery 2005 and 2006. Int J Colorectal Dis. 2008;23:901-908.

6. Naef M, Naef U, Mouton W, et al. Outcome and complications after laparoscopic Swedish adjustable gastric banding: 5-year results of a prospective clinical trial. Obes Surg. 2007;17: 195-201.
7. Weiner R, Blanco-Engert R, Weiner S, et al. Outcome after laparoscopic adjustable gastric banding -8 years experience. Obes Surg. 2003;13:427-434.

8. Meir E, Van Baden M. Adjustable silicone gastric banding and band erosion: personal experience and hypotheses. Obes Surg. 1999;9:191-193.

9. Lattuada E, Zappa MA, Mozzi E, et al. Histologic study of tissue reaction to the gastric band: does it contribute to the problem of band erosion? Obes Surg. 2006;16:1155-1159.

10. Forsell P, Hallerbäck B, Glise H, et al. Complications following Swedish adjustable gastric banding (SAGB) — a long-term follow-up. Obes Surg. 1999;9:11-15.

11. Singhal R, Bryant C, Kitchen M, et al. Band slippage and erosion after laparoscopic gastric banding: a meta-analysis. Surg Endosc. 2010;24:2980-2986.

12. O'Brien PE, Dixon JB, Laurie C, Anderson M. A prospective randomized trial of placement of the laparoscopic adjustable gastric band: comparison of the perigastric and pars flaccid pathways. Obes Surg. 2005;15(6):820-826.

13. Di Lorenzo N, Furbetta F, Favretti F, et al. Laparoscopic adjustable gastric banding via pars flaccida versus perigastric positioning: technique, complications, and results in 2,549 patients. Surg Endosc. 2010;24(7):1519-1523.

14. Mozzi E, Lattuada E, Zappa MA, et al. Treatment of band erosion: feasibility and safety of endoscopic band removal. Surg Endosc. 2011;25(12):3918-3922.

15. Cherian PT, Goussous G, Ashori F, Sigurdsson A. Band erosion after laparoscopic gastric banding: a retrospective analysis of 865 patients over 5 years. Surg Endosc. 2010;24:20312038.

16. Mittermair RP, Obermüller S, Perathoner A, Sieb M, Aigner F, Margreiter R. Results and complications after Swedish adjustable gastric banding-10 years experience. Obes Surg. 2009;19(12): 1636-1641.

17. Kohn GP, Hansen CA, Gilhome RW, McHenry RC, Spilias DC, Hensman C. Laparoscopic management of gastric band erosions: a 10-year series of 49 cases. Surg Endosc. 2012;26(2): $541-545$. 